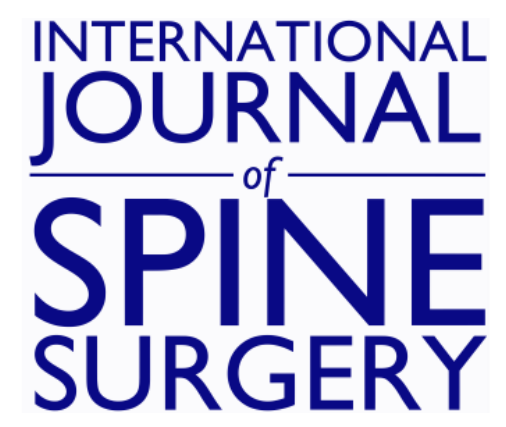

\title{
Outcome Measures of an Intracanal, Endoscopic Transforaminal Decompression Technique: Initial Findings from the MIS Prospective Registry
}

Joseph A. Sclafani, Kamshad Raiszadeh, Dan Laich, Jian Shen, Matthew Bennett, Robert Blok, Kevin Liang and Choll W. Kim

Int J Spine Surg 2015, 9 ()

doi: https://doi.org/10.14444/2069

http://ijssurgery.com/content/9/69

This information is current as of April 25, 2023.

Email Alerts Receive free email-alerts when new articles cite this article. Sign up at:

http://ijssurgery.com/alerts

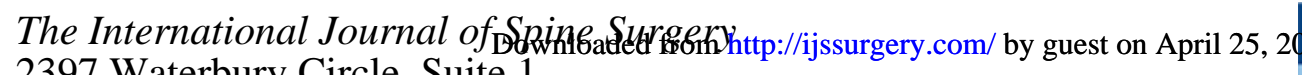
2397 Waterbury Circle, Suite 1,

Aurora, IL 60504, Phone: +1-630-375-1432 


\section{Outcome Measures of an Intracanal, Endoscopic Transforaminal Decompression Technique: Initial Findings from the MIS Prospective Registry}

Joseph A. Sclafani, MD, 1,2 Kamshad Raiszadeh, MD, 1 Dan Laich, DO, ${ }^{3}$ Jian Shen, MD, PhD, ${ }^{4}$ Matthew Bennett, MD, 5 Robert Blok, DO, 6 Kevin Liang, PhD, ${ }^{2}$ Choll W. Kim, MD, $P h D^{1}$

${ }^{1}$ Spine Institute of San Diego, San Diego, CA, 2Milestone Research Organization, San Diego, CA, ${ }^{3}$ Swedish Covenant Hospital, Chicago, IL, ${ }^{4}$ Mohawk Valley Orthopedics, Amsterdam, NY, ${ }^{5}$ Tier Orthopedic Associates, Johnson, NY, ${ }^{6}$ Clark Memorial Medical Center, Clarksville, IN

\section{Abstract}

Background

Minimally invasive transforaminal endoscopic procedures can achieve spinal decompression through either direct or indirect techniques. Subtle variations in trajectory of the surgical corridor can dictate access to the pathologic tissue. Two general strategies exist: the intradiscal "inside-out" technique and the extradiscal, intracanal (IC) technique. The IC technique utilizes a more lateral transforaminal approach than the intradiscal technique, which allows for a more direct decompression of the spinal canal.

\section{Objective}

This study is an assessment of IC patient outcome data obtained through analysis of a previously validated MIS Prospective Registry.

\section{Methods}

Post-hoc analysis was performed on the MIS Prospective Registry database containing 1032 patients. A subgroup of patients treated with the endoscopic IC technique was identified. Patient outcome measures after treatment of symptomatic disk herniation and neuroforaminal stenosis were evaluated.

\section{Results}

A total of 86 IC patients were analyzed. Overall, there was significant improvement in employment and walking tolerance as soon as 6 weeks post-op as well as significant one year VAS and ODI score improvement. Subanalysis of IC patients with two distinct primary diagnoses was performed. Group IC-1 (disc herniation) showed improvement in ODI and VAS back and leg outcomes at 1 year post-op. Group IC-2 (foraminal stenosis) showed VAS back and leg score improvement at one year post-op but did not demonstrate significant improvement in overall ODI outcome at any time point. The one year re-operation rate was $2 \%(1 / 40)$ for group IC- 1 and $28 \%(5 / 18)$ for group IC-2.

\section{Conclusions}

The initial results of the MIS Registry IC subgroup show a significant clinical improvement when the technique is employed to treat patients with lumbar disc herniation. The treatment of foraminal stenosis can lead to improved short-term clinical outcome but is associated with a high re-operation rate at 1 year post-op.

KEYWORDS: MINIMALLY INVASIVE SPINE SURGERY, ENDOSCOPIC DECOMPRESSION, STENOSIS, DISC HERNIATION, CLINICAL OUTCOME VOLUME 9 ARTICLE 69 DOI: 10.14444/2069

\section{Introduction}

Minimally invasive spine (MIS) procedures avoid extensive soft tissue dissection and disruption of the stabilizing paraspinal musculature, which can substantially decrease post-operative morbidity and im- prove health care economics. ${ }^{1}$. There has been an increasing demand for less invasive surgical options by both patients and referring physicians as evidence for the utility and benefit of MIS techniques continues to propagate. Subsequently, there has been a surge of innovation and development of novel minimally inva- 
sive surgical approaches to address spinal disorders after conservative measures fail.

Minimally invasive lumbar discectomy has significantly evolved since being introduced by Kambin and Gellman in $1983^{2}$. Initially, these procedures achieved decompression through a central nucleotomy to indirectly relieve pressure on the exiting nerve root. ${ }^{2,3}$ Frost and Hausmann later proposed the concurrent use of intervertebral endoscopy with intradiscal nucleotomy to visually confirm adequate decompression about the nerve root. ${ }^{4}$ These endoscopic procedures utilized an intradiscal method commonly referred to as the "inside-out technique" that began with a partial nucleotomy followed by removal of disc fragments through the nucleotomy defect and endoscopic channel. ${ }^{5}$ The "inside-out" technique has been shown to be highly effective, but surgeon adoption rate has been impacted by the lengthy and gradual learning curve associated with this technically demanding procedure. ${ }^{6}$

The intracanal endoscopic (IC) technique is an extradiscal approach that utilizes a more cephalad to caudad approach through the neuroforamen to allow direct removal of the disc herniation from within the canal (Figure 1).$^{7-9}$ This study utilizes a validated, prospective registry to compare the effectiveness of the intracanal (IC) endoscopic technique to treat lumbar disk herniations vs. foraminal stenosis.

\section{Methods}

Analysis was performed on the MIS Prospective Registry, a previously validated prospective, multicenter database established to collect Health Related Quality of Life (HRQOL) outcome metrics on patients treated with various minimally invasive spine surgery techniques. ${ }^{10}$ Patient data obtained from 17 surgeons at 15 sites of various practice settings were de-identified and electronically entered into the MIS Registry. Patient assessment of Oswestry Disability Index (ODI) and Visual Analog Scale (VAS) for low back and radiating leg pain were obtained at the preop, 2 week, 6 week, 3 month, 6 month, 12 month and 24 month post-operative time points. A novel preoperative diagnosis and surgical procedure table was constructed to allow data collection on a level-by- level basis for each patient. These diagnosis and procedure grids were completed by the operating surgeon and electronically entered into the Registry database. Complications were collected through a distinct, surgeon-completed questionnaire. Reportable complications were collected using the Spine Adverse Events Severity System. ${ }^{11}$

Post-hoc analysis was performed on Registry data to extract subjects who underwent IC procedures using a single decompression system (Joimax Inc., Irvine, CA, USA). The IC endoscopic technique utilizes a transforaminal, extra-discal approach previously described by Hoogland and recently modified by Ipren-

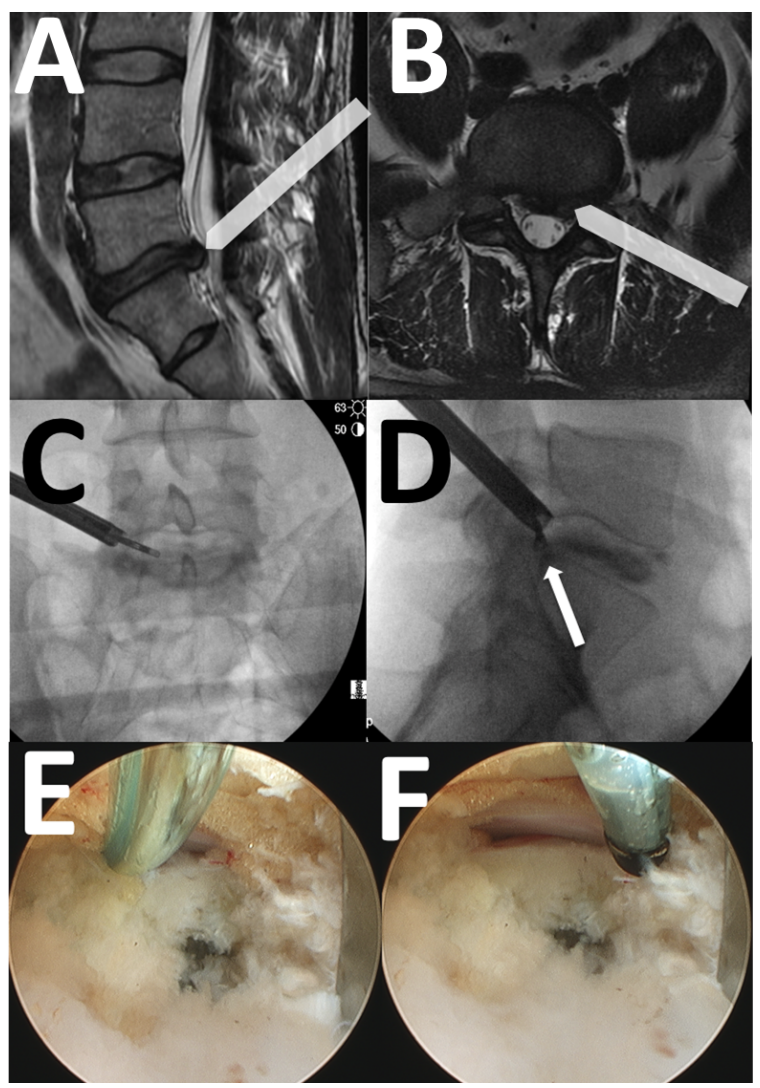

Fig. 1. A) Sagittal T2 image of the lumbar spine showing an L5-S1 disk herniation. The arrow-bar shows the trajectory of the endoscopic cannula in the cephalad-caudal direction. B) T2 Axial image of the L5-S1 level showing a left-sided disk herniation. The arrow-bar shows the trajectory of the endoscopic cannula in the lateral-medial direction. C) Antero-posterior $c$-arm image showing the curved grasper reaching toward the disk herniation. D) Lateral c-arm image of the same patient showing the grasper at the posterior disk margin, corresponding to the location of the disk herniation (white arrow). E) Intraoperative photograph with the flexible curved probe (Trigger-flex, Elliquence, Baldwin, New York) inserted between the traversing nerve and the posterior annulus. F) Intraoperative photograph of the Ellman probe pulled back, showing the lateral edge of the traversing nerve root/dural tube. 
burg. ${ }^{12,13}$

Compared to the intradiscal "inside-out" technique, the IC approach trajectory is more cephalad and lateral in an effort to reach the disk herniation within the canal (Figure 1A and Figure 1B). External reamers of increasing diameter are used to open the neuroforamen, which allows the endoscopic cannula to reach the spinal canal without violation of the posterior annulus. Using various angled instruments, including a flexible, curved grasper, the disk fragments are removed directly (Figure $1 \mathrm{C}$ and Figure 1D).

Data were segmented based on gender, primary indication, operative level, previous lumbar surgery, BMI, co-morbidity and pre-operative pain score for outcome measure analysis of each subgroup. Specific questions within the ODI questionnaire were evaluated to assess post-operative change in walking tolerance (ODI question \#4) and employment limitations (ODI question \#8). Statistical analyses were performed via one-way ANOVA or t-tests with significance defined as $\mathrm{p}<0.05$. Data are reported as means $\pm \mathrm{SD}$, unless otherwise specified.

\section{Results}

A total of 86 IC patients with a median age of 49 years (range 18-89 years) were extracted from the total MIS Prospective Registry population of 1032 patients for analysis (Figure 2). Median hospital stay was 0 days. Overall ODI scores were $43.4 \pm 18.0$ preop $(\mathrm{n}=83), 29.4 \pm 19.7$ at 6 weeks post-op $(\mathrm{n}=45$, $\mathrm{p}=0.001), 24.6 \pm 21.4$ at 6 Months post-op $(\mathrm{n}=23$, $\mathrm{p}=0.001)$ and $25.4 \pm 23.1$ at 1 year post-op $(\mathrm{n}=59$, $\mathrm{p}=0.001$ ) (Figure 3). Employment disability showed significant improvement at all post-op time points: Pre-operative $(3.3 \pm 1.3, \mathrm{n}=83), 6$ week post-op $(2.2 \pm$ $1.3, \mathrm{n}=45, \mathrm{p}=0.001), 3$ month post-op $(1.9 \pm 1.2, \mathrm{n}=$ $37, \mathrm{p}=0.001), 6$ month post-op $(1.7 \pm 1.4, \mathrm{n}=27$, $\mathrm{p}=0.001)$, and 1 year post-op $(1.2 \pm 1.1, \mathrm{n}=59$, $\mathrm{p}=0.001)$. Walking disability also improved at all post-operative time points: Pre-operative $(2.9 \pm 1.3$, $\mathrm{n}=82), 6$ week post-op $(2.0 \pm 1.4, \mathrm{n}=47, \mathrm{p}=0.01), 3$ month post-op $(2.0 \pm 1.5, \mathrm{n}=45, \mathrm{p}=0.01), 6$ month post-op $(1.9 \pm 1.5, \mathrm{n}=27, \mathrm{p}=0.05)$, and 1 year post-op $(1.6 \pm 1.4, n=59, p=0.001)$ (Figure 4). There was significant overall VAS score improvement: pre- operative VAS (back=5.9 \pm 2.8, leg $=6.6 \pm 2.6, \mathrm{n}=78)$, 6 week post-op $($ back $=3.2 \pm 2.9$, leg $=3.4 \pm 2.9, \mathrm{n}=49$, $\mathrm{p}<0.05), 6$ month post-op (back=2.8 \pm 2.8, leg=2.5 \pm $2.7, \mathrm{n}=24, \mathrm{p}<0.05)$, and 1 year post-op (back=3.1 \pm 2.9, leg $=1.7 \pm 2.2, \mathrm{n}=60, \mathrm{p}<0.05$ )(Figure 3 ).

There were no intraoperative or perioperative complications such as dural tears, nerve injuries, postoperative infections, or 90 -day readmissions.

A subanalysis of IC patients with two distinct primary diagnoses was performed. Group IC-1 underwent treatment for disc herniation with radiculopathy $(n=40)$. The primary indication for group IC-2 was bony neuroforaminal stenosis secondary to spondylosis or a stable grade 1 spondylolisthesis $(\mathrm{n}=18)$ (Figure 2). A diagnosis of spondylolisthesis did not result

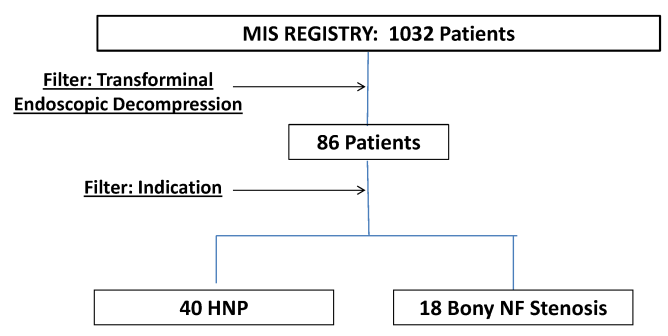

Fig. 2. Schematic demonstrating Post-hoc extraction of IC patients from the MIS registry.

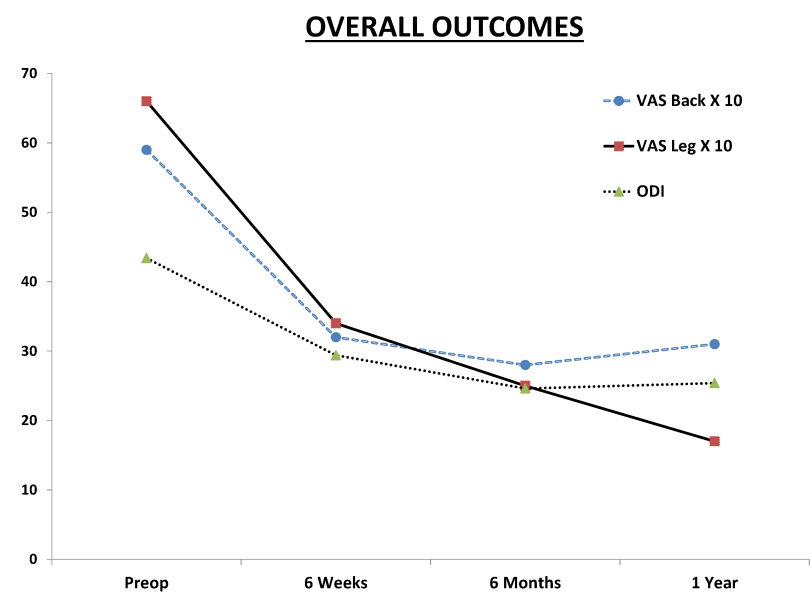

Fig. 3. Improvement in Oswestry Disability Index score, VAS back and leg pain score by time point for the overall IC population. 
in significant differences between any outcome measures when compared to subjects with spondylosis. Group IC-1 (disc herniation) showed improvement in ODI (19.4 points, $\mathrm{p}=0.0002, \mathrm{n}=40$ ) and VAS (back$=2.9$ points, leg $=2.9$ points, $\mathrm{p}=0.0001, \mathrm{n}=40$ ) scores at 1 year post-op. There was also significant improvement in functional walking ability ( 1.5 points, $\mathrm{n}=36$, $\mathrm{p}<0.0001)$ and employment tolerance (2.6 points, $\mathrm{n}=36, \mathrm{p}<0.0001$ ) at one year post-op. Group IC-2 (foraminal stenosis) showed VAS score improvement (back=2.7 points, leg $=3.5$ points, $\mathrm{p}=0.001, \mathrm{n}=18$ ) at one year post-op but did not demonstrate significant improvement in overall ODI outcome (7.1 point improvement, $\mathrm{p}=0.06, \mathrm{n}=17)$. Subjects that underwent intracanal endoscopic decompression for a primary diagnosis of bony foraminal stenosis did demonstrate significant improvement in walking tolerance (1.1 points, $n=17, p=0.003$ ) and employment tolerance (1.2 points, $n=17, \mathrm{p}=0.005$ ) at one year post-op. One year re-operation rate was $2 \%(1 / 40$, a revision endoscopic discectomy for recurrent disk herniation) for group IC-1 and 28\% (5/18) for group IC-2. The 5 reoperations in group IC-2 involved subsequent reconstruction and fusion for definitive treatment.

\section{Discussion}

This analysis of the MIS Prospective Registry intracanal transforaminal endoscopic lumbar surgery subgroup demonstrates significant clinical improvement lasting from the initial perioperative period to at least 1 year after surgery. There was clinical improvement

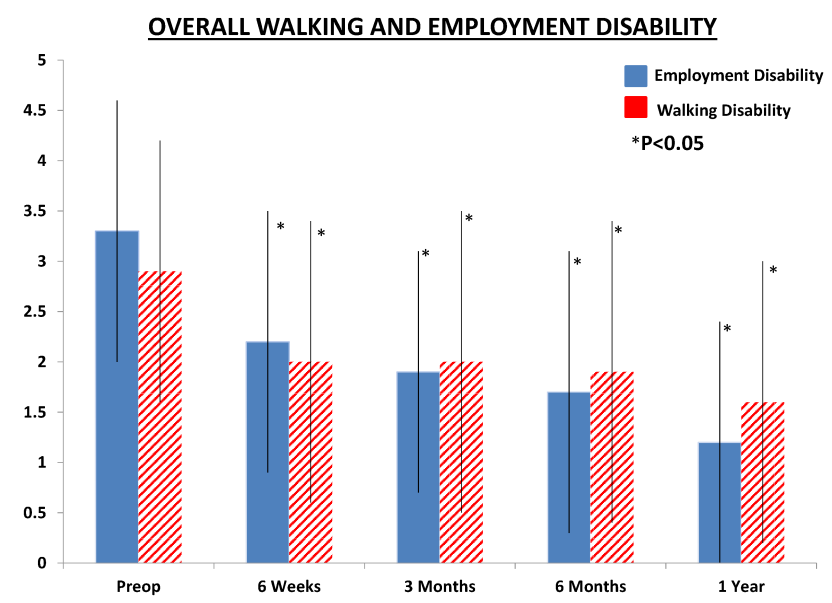

Fig. 4. Improvement in Employment Tolerance (ODI question \#8) and walking tolerance score (ODI question \#4) by time point for the overall IC population. in axial low back pain, leg pain, and key functional parameters of the Oswestry Disability Index in all analyzed subgroups. The endoscopic technique revealed a high safety profile, with all patients successfully treated on an outpatient basis without an intraoperative complication or 90 day readmission.

Stratification of data based on primary surgical indication demonstrated the best patient outcomes occurred after IC endoscopic decompression of a soft disc herniation with radiculopathy. This subgroup of patients reported functional improvement surpassing the minimal clinically important difference in Oswestry Disability Index score as well as increased employment tolerance starting 6 weeks after surgery. ${ }^{14}$

The clinical improvement after IC endoscopic decompression is comparable to what has been reported in many studies of microdiscectomy, which remains the current "gold standard" surgical decompression technique. ${ }^{15-21}$ This is best displayed in the IC-1 group that demonstrated a 19.4 point improvement in ODI and a re-operation rate of $2 \%$ at one year. Improvement in ODI after microdiscectomy to treat a soft disc herniation has been reported to range from 6-8 points to over 30 points in studies such as the SPORT randomized trial. ${ }^{15,17,21}$ Findlay et al. reported a $5.1 \%$ rate of revision surgery in a 10 year follow-up study after microdiscectomy while others, such as Kowalski et al., report re-operation rates as high as $12 \%$ at 34 months after a primary microdiscectomy. ${ }^{20,21} \mathrm{~A}$ limitation of our study is the lack of long-term outcome data that can be used to calculate the rate of symptomatic degenerative disc disease at 5-10 years after surgery. However, the reoperation rate for a recurrent disc herniation one year after IC decompression of a soft disc herniation was $1.8 \%$, which is similar to the $2 \%$ one-year reoperation rate for recurrent disc herniation that was reported in the often cited SPORT trial. ${ }^{15}$

The treatment of bony foraminal stenosis with endoscopic decompression can lead to improved short term clinical outcome but is associated with a high rate of revision arthrodesis procedures within one year. This is likely secondary to technical limitations inherent to transforaminal endoscopic decompres- 
sion systems. Namely, the osseous reaming apparatus may result in inadequate intraoperative decompression of osseous structures leading to residual symptoms in some patients within this subgroup. Newly developed intracanal, endoscopic decompression systems have implemented novel features to address osseous foraminal stenosis including a less cumbersome reaming tool that can be manipulated within the endoscope with more agility by the operating surgeon.

The main limitation of this study is the inconsistent rate of data collection at scheduled follow up intervals, including the one year follow up period. Although data collection through a prospective registry allows post-hoc extraction of a large sample size, there is inherently less stringent monitoring of patient data collection than with a randomized controlled trial. Additionally, this study did not record the duration of symptoms prior to surgical intervention and did not include a non-surgical control group. Nevertheless, this study of endoscopic transforaminal discectomy demonstrates promising results in patients with symptomatic disc herniations.

\section{Complications}

There were no reported complications including dural tears, neurovascular injuries, post-operative infections, medical sequele or 90 day readmissions.

\section{Conclusion}

This study shows a significant clinical and functional improvement when the IC endoscopic technique is employed to treat patients with lumbar disc herniation. The treatment of foraminal stenosis can lead to improved short-term clinical outcome but is associated with a high re-operation rate at 1 year postop. The long-term outcomes, together with higher-level analysis of cost-effectiveness (cost/quality adjusted life year) will be forthcoming with the continued growth of the MIS Registry database.

\section{References}

1. Allen RT, Garfin SR. The economics of minimal- ly invasive spine surgery: the value perspective. Spine, 35(26S), S375-S382, 2010.

2. Kambin P, Gellman H. Percutaneous lateral discectomy of the lumbar spine. A preliminary report. Clinical Orthopaedics, 174,127-132, 1983.

3. Hijikata S, Yamagishi M, Nakayma T. Percutaneous discectomy: a new treatment method for lumbar disc herniation. J Tokyo Den-ryoku Hosp, 5: 39-44, 1975.

4. R Forst, G Hausmann. Nucleoscopy-a new examination technique. Arch Orthop Traum Surg, 101: 219-221, 1983.

5. Yeung AT, Tsou PM. Posterior endoscopic excision for lumbar disc herniation: Surgical technique, outcome, and complications in 307 consecutive cases. Spine, 27(7): 722-731, 2002.

6. Morgenstern R, Morgenstern C, Yeung, AT. The learning curve in foraminal endoscopic discectomy: experience needed to achieve a $90 \%$ success rate. SAS journal, 1(3): 100-107, 2007.

7. Tsou PM, Yeung AT. Transforaminal endoscopic decompression for radiculopathy secondary to intracanal noncontained lumbar disc herniations: outcome and technique. The Spine Journal, 2(1): 41-48, 2002.

8. Mathews HH. Transforaminal endoscopic microdiscectomy. Neurosurg Clin N Am, 7: 59-63, 1996.

9. Ruetten S, Komp M, Godolias G. An extreme lateral access for the surgery of lumbar disc herniations inside the spinal canal using the full-endoscopic uniportal transforaminal approach-technique and prospective results of 463 patients. Spine, 30(22):2570-2578, 2005.

10. Sclafani J, Raiszadeh K., Raiszadeh R., Kim P, Doerr T, Siddiqi F, Kim C. Validation and Analysis of a Multi-site MIS Prospective Registry Through Sub-analysis of an MIS TLIF Subgroup. International Journal of Spine Surgery, 8(1), 4-4, 2014.

11. Ramersaud YR, Neary MA, White K. Spine adverse events severity system. Content validation and interobserver reliability assessment. Spine.

2010;35:790-795.

12. Hoogland T. Transforaminal endoscopic discectomy with foraminoplasty for lumbar disc herniation. Surg Tech Orthop Traumatol. 40, 55-120, 2003.

13. Iprenburg M. Transforaminal endoscopic 
surgery - technique and provisional results in primary disc herniation. Eur Musculoskelet Rev. 2007, 73-76, 2007.

14. Bombardier C. Outcome assessments in the evaluation of treatment of spinal disorders: Summary and general recommendations. Spine, 25:3100-3103, 2000.

15. Weinstein JN, Tosteson TD, Lurie JD, et al. Surgical vs nonoperative treatment for lumbar disk herniation: the Spine Patient Outcomes Research Trial (SPORT) A Randomized Trial. JAMA, 296(20):2441-2450, 2006.

16. Asch HL, Lewis PJ, Moreland DB, Egnatchik JG, Yu YJ, Clabeaux DE, Hyland AH. Prospective multiple outcomes study of outpatient lumbar microdiscectomy: Should 75 to $80 \%$ success rates be the norm? J Neurosurg 96 [Suppl 1]:34-44, 2002. 17. Carragee EJ, Han MY, Suen PW, Kim D. Clinical outcomes after lumbar discectomy for sciatica: The effects of fragment type and anular competence. J Bone Joint Surg Am 85A:102-108, 2003.

18. Daneyemez M, Sali A, Kahraman S, Beduk A, Seber N. Outcome analyses in 1072 surgically treated lumbar disc herniations. Minim Invasive Neurosurg 42:63-68, 1999.

19. Davis RA. A long-term outcome analysis of 984 surgically treated herniated lumbar discs. J Neuro- surg 80:415-421, 1994.

20. Findlay GF, Hall BI, Musa BS, Oliveira MD, Fear SC. A 10-year follow-up of the outcome of lumbar microdiscectomy. Spine 23:1168-1171, 1998.

21. Kowalski JM, Olsewski JM, Simmons ED Jr. Results of intervertebral diskectomy without fusion at L4-5 versus L5-S1. J Spinal Disord 8:457-463, 1995.

\section{Disclosures}

The MIS prospective registry was funded by multiple sponsors, one of which was Joimax. Additionally, more than one author of this paper discloses that they are a consultant for Joimax Inc. (CWK, DL, JS).

\section{Corresponding Author}

Choll W. Kim, MD PhD, Spine Institute of San Diego, 6719 Alvarado Road, Ste 308, San Diego, CA 92120. choll@siosd.com.

Published 11 December 2015.

This manuscript is generously published free of charge by ISASS, the International Society for the Advancement of Spine Surgery. Copyright @ 2015 ISASS. To see more or order reprints or permissions, see http://ijssurgery.com. 